\title{
B7 liquid crystal filament growth in the presence of carbon nanotubes
}

DOI:

10.1002/cphc.201800949

$10.1002 /$ cphc.201800949

\section{Document Version}

Accepted author manuscript

Link to publication record in Manchester Research Explorer

\section{Citation for published version (APA):}

Stamatoiu, O., \& Dierking, I. (2018). B7 liquid crystal filament growth in the presence of carbon nanotubes.

ChemPhysChem. https://doi.org/10.1002/cphc.201800949, https://doi.org/10.1002/cphc.201800949

\section{Published in:}

ChemPhysChem

\section{Citing this paper}

Please note that where the full-text provided on Manchester Research Explorer is the Author Accepted Manuscript or Proof version this may differ from the final Published version. If citing, it is advised that you check and use the publisher's definitive version.

\section{General rights}

Copyright and moral rights for the publications made accessible in the Research Explorer are retained by the authors and/or other copyright owners and it is a condition of accessing publications that users recognise and abide by the legal requirements associated with these rights.

\section{Takedown policy}

If you believe that this document breaches copyright please refer to the University of Manchester's Takedown Procedures [http://man.ac.uk/04Y6Bo] or contact uml.scholarlycommunications@manchester.ac.uk providing relevant details, so we can investigate your claim.

\section{OPEN ACCESS}




\section{Accepted Article}

Title: B7 liquid crystal filament growth in the presence of carbon nanotubes

Authors: Ingo Dierking and O. Stamatoiu

This manuscript has been accepted after peer review and appears as an Accepted Article online prior to editing, proofing, and formal publication of the final Version of Record (VoR). This work is currently citable by using the Digital Object Identifier (DOI) given below. The VoR will be published online in Early View as soon as possible and may be different to this Accepted Article as a result of editing. Readers should obtain the VoR from the journal website shown below when it is published to ensure accuracy of information. The authors are responsible for the content of this Accepted Article.

To be cited as: ChemPhysChem 10.1002/cphc.201800949

Link to VoR: http://dx.doi.org/10.1002/cphc.201800949 


\section{Graphical abstract}

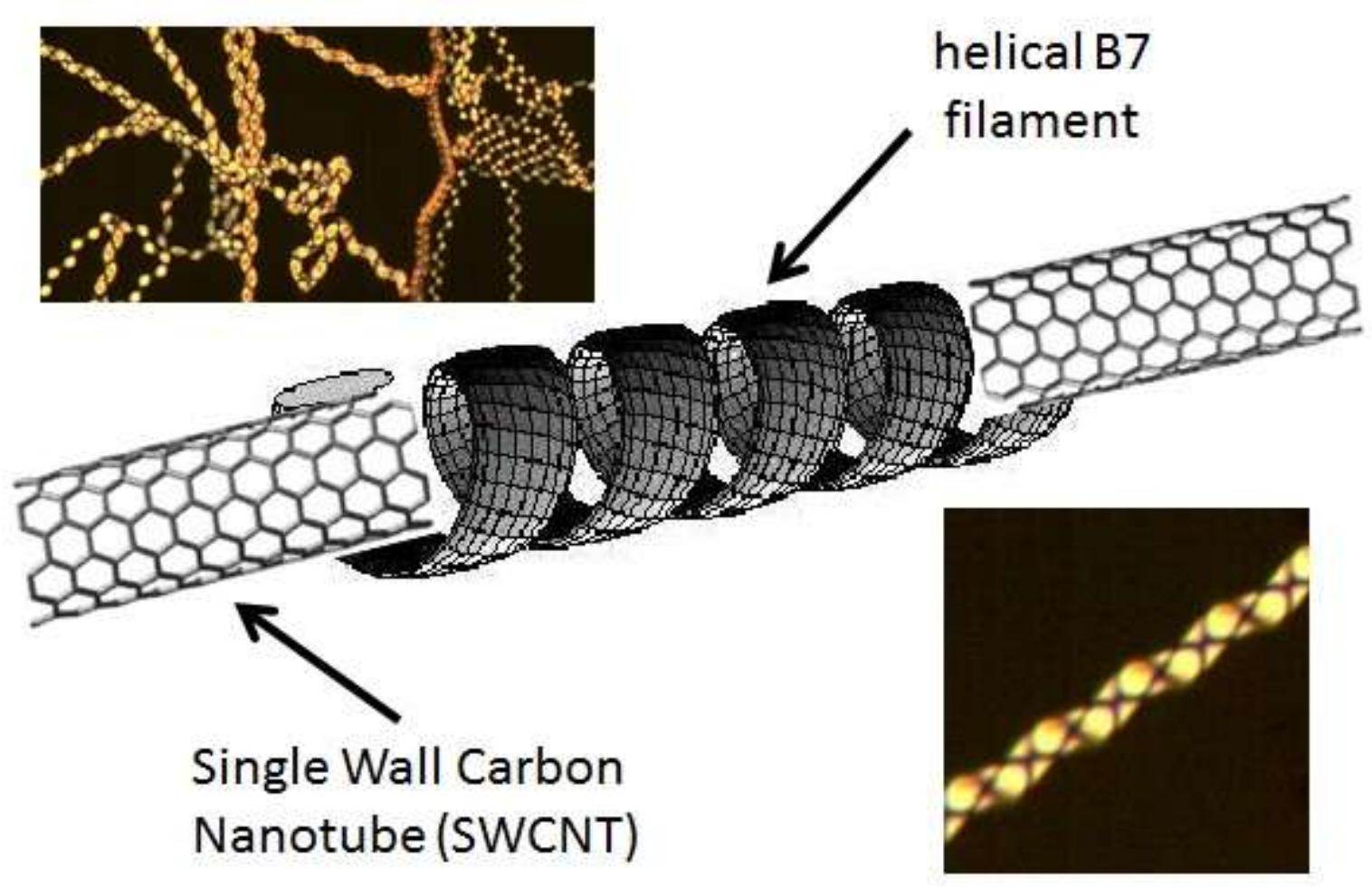

The B7 phase of bent-core molecules is one of the most intriguing and enigmatic of the liquid crystal phases. The helical growth of filament structures is investigated in the presence of single wall carbon nanotubes, which act as a core for the helix, similar to the helix-rod of a piston filler. Buckling phenomena is described and possible applications discussed. 


\title{
B7 liquid crystal filament growth in the presence of carbon nanotubes
}

\author{
Dr O. Stamatoiu ${ }^{1,2}$, Dr I. Dierking ${ }^{1 *}$ \\ ${ }^{1}$ School of Physics and Astronomy, University of Manchester, Oxford Road, Manchester \\ M139PL, United Kingdom \\ 2 Faculty of Physics, West University of Timisoara, 4 Vasile Pârvan Str., Timisoara, 300223, \\ Romania
}

* author for correspondence, electronic mail: ingo.dierking@manchester.ac.uk

Keywords: liquid crystal, nanotubes, bent-core mesogens, B7 phase, filament growth, buckling instability

\section{Abstract}

Liquid crystal phases formed from bent-core mesogens have attracted much interest of the liquid crystal research community, due to the manifestation of chirality effects from achiral molecules. One of the most elusive of the bent-core phases is the B7 phase, which at its early stage often forms in a helical filament fashion. We investigate the growth of such filaments in the presence of single-walled nanotubes to elucidate possible effects on the growth dynamics and helicity of B7 helical filaments. It is found that the filament width slightly decreases in comparison to the neat B7 material, suggesting a more tightly bound structure around the nanotubes, with the nanotubes likely acting as the core of the helical filament. No effects on pitch or periodicity of the helical superstructure is observed. The filament growth velocity quickly decreases as nanotubes are added to the B7 phase, indicating that a more tightly bound structure needs a longer time of formation. An observed buckling instability is of interest as a microscopic example for the study of nonlinear dynamics theories of filaments. The present investigation is thus of general 
importance for nanoparticle directed growth of filaments, which has applications in biomolecular growth and high tensile strength fibres. 


\section{Introduction}

Liquid crystals[1-3] are anisotropic fluids, which are thermodynamically located between the isotropic liquid and the three dimensionally ordered crystal. These partially ordered phases are observed for shape-anisotropic molecules, which can be rod-like (calamitic), disc-like (discotic), or banana-shaped thus of bent-core nature and is reflected in the anisotropy of the liquid crystal phases through the molecular packing. Many different liquid crystalline phases have been described, ranging from those with purely orientational order, called nematic, to those with additional one- or two-dimensional positional order, called smectic, in the case of rod- and bent-shaped molecules.

Since their discovery[4], bent-core liquid crystals [5-7] have proven to be a very fruitful ground for fundamental and applied research investigations of liquid crystal phases. This is mainly due to the observation that with these materials, despite the fact that their constituent molecules are achiral, chiral structures and effects can be detected. For example, the addition of achiral bent-core molecules to a helical phase can amplify chirality and thus decrease the pitch. Also, spontaneous chiral segregation into optically left- and right-handed domains has been observed[8], as well as an increase of chirality for ferroelectric liquid crystals[9-11]. At least seven principle bent-core liquid crystalline phases have been discovered so far, of which the B7 phase is probably the most intriguing and most complicated at the same time. On cooling from the isotropic melt, a wealth of structural features can be identified by polarizing microscopic observation, such as the formation of left-and right-handed helices, and single and doubly twisted ribbons [12-15], similar to normal and super-twisted telephone cords, but also domains with equidistant striped patterns which indicate some twisted, or undulated structures [12, 15-18].

During the same time period as the discovery of bent-core phases, also dispersions of liquid crystal phases, generally simple nematics, with spherical and elongated nanoparticles have increasingly attracted interest[19]. The latter were at first dielectric and ferroelectric nanoparticles, which were added to nematic liquid crystals in order to tune and improve the materials properties for applications in the area of displays and non-display devices. Later, nanotubes were added to nematics in order to align the nanotubes through interaction with 
the liquid crystal host and to exploit the electric conduction properties of the nanotubes for novel device materials[20,21].

In this paper, we investigate the effect of single wall carbon nanotubes (SWCNT) on the formation, growth dynamics and possible structure of the B7 phase filaments when they form from the isotropic melt. The reasoning behind this study is the likeliness that the nanotubes will act as seed particles for the filament formation, and that the helical growth will take place with B7 smectic layers wrapping around the nanotubes, such that the latter will take the role of the filament core. With the demonstration that B7 filaments can be directed to grow into a uniform direction by the use of confining channels [16], this could provide an elegant mechanism to exploit liquid crystal self-organization to produce uniform arrays of nanotubes, and thus open the possibility for switchable one-dimensional conducting devices.

\section{Experimental}

The bent-core material used in this study is a typical banana-shaped compound in which the rigid core is composed of five phenyl rings connected through ester and azomethin groups, while the flexibility originates from the two $n$-decyloxy chains grafted in para position on the terminal aromatic rings. It was provided by the group of G. Heppke, Berlin, and has the following structural formula [22]:

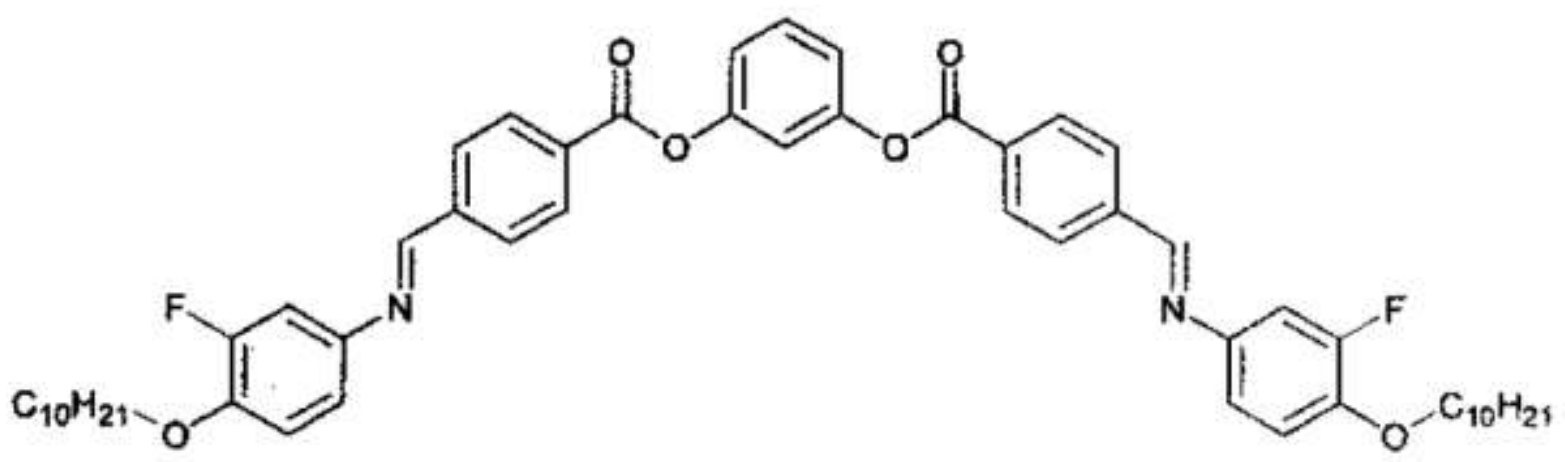

Its phase sequence on heating is given by Cryst. 119 B7 159 Iso., while it was observed as Iso. 161 B7 96 Cryst. on slow cooling, as determined by polarizing optical microscopy (Leica 
OPTIPHOT), equipped with a Linkam hot stage (LTS350) and temperature controller (TP94) for relative temperature stability to $0.1 \mathrm{~K}$. No appreciable or systematic changes in phase transition temperatures were observed through the addition of nanotubes. The single wall carbon nanotubes (SWCNT) employed, were commercially available from CarboLex at a purity of $99.5 \%$ and used as received, without any further purification or treatment. The major impurities are carbon nanospheres (fullerenes) and carbon-encapsulated metallic catalyst nanoparticles. The single walled nanotube diameters were approximately 1.2$1.5 \mathrm{~nm}$. These are known to form bundles of tubes due to van der Waals interactions. From scanning electron microscopy (SEM) it is known that the average bundle diameter is about $60 \mathrm{~nm}[20]$, which is consistent with the order of magnitude size of liquid crystal defect cores. Samples at varying concentrations between $0-0.2 \%$ by weight nanotube content were prepared by dissolving an amount of nanotubes in dichloromethane and adding the respective amount of dissolved nanotubes to the liquid crystal. The then isotropic solution of solvent, liquid crystal and nanotubes was thoroughly stirred and subjected to $1 \mathrm{~h}$ of mild ultrasound treatment in a water bath at $40 \mathrm{kHz}$, until all the solvent had evaporated. This could be confirmed through measurements of the clearing temperatures, which stayed practically constant within experimental error. Commercial cells (AWAT, Poland) of thickness $10 \mu \mathrm{m}$ and planar alignment layers, were capillary filled and investigated by microscopy. For the investigations samples were cooled at a rate of $0.1 \mathrm{Kmin}^{-1}$, which was determined to be sufficient to generate reproducible results, and which lies within the limits of error of the available experimental equipment. The isotropic to B7 phase transition temperature was taken to be the temperature at which first filaments could be observed. For reasons of comparability, all measurements on individual samples with different concentrations were carried out at the same reduced temperature of $\mathrm{T}_{\text {Iso- } \mathrm{B7}}-\mathrm{T}=0.2 \mathrm{~K}$ (or quench depth of $0.1 \mathrm{~K}$ ), which minimizes effects of supercooling between different measurement series. Movies of the growth process were acquired at a frame rate of $14 \mathrm{fps}$ at a resolution of $2048 \times 1088$ pixels for further analysis (microscope mounted uEye digital camera). Individual series of image frames were extracted and subsequently analysed using software ImageJ. 


\section{Results and Discussion}

\subsection{Filament growth}

Typical polarizing micrographs of the B7 phase growing from the isotropic melt are depicted in figure 1. In part (a) a complete texture is shown, with single and double helical filaments, similar to twisted and super-twisted telephone cords, as well as conformal domains which show equidistant line patterns of areas with periodic layer undulations.
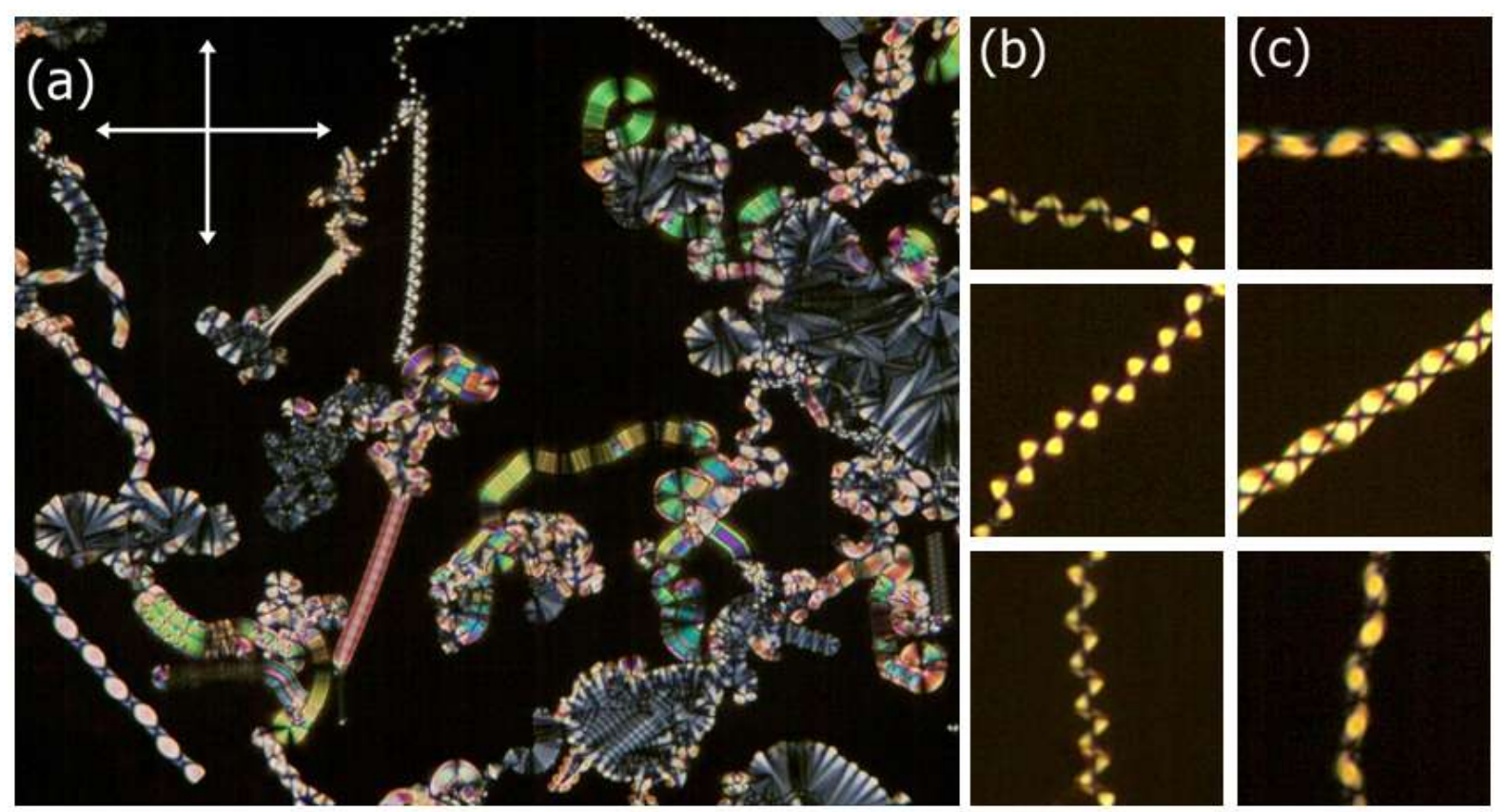

Figure 1: (a) texture of the B7 phase growing from the black background of the isotropic melt. The crossed polarizers are indicated and the height of the photograph is approximately $500 \mu m$. The images of columns (b) and (c) show close-ups of single twisted and double twisted filaments, respectively, at orientations of $0^{\circ}, 45^{\circ}$ and $90^{\circ}$ with respect to the crossed polarizers. The scale is about twice as large as for the texture of part (a).

Also to be seen are areas with focal conic domains and leaf-like domains. There is thus a hierarchy and variation of structures and patterns to be observed, which is common for the B7 phase and which has been studied in detail by Deb et al.[15], with polarized optical microscopy (POM) as well as fluorescence confocal polarizing microscopy (FCPM). For reasons of illustration and clarity we have also added enlarged images of single- (part (b)) 
and double-twisted (part (c)) filaments in orientations to the crossed polarizers of $0^{\circ}, 45^{\circ}$ and $90^{\circ}$, because these will be of the prime interest for this investigation. It is clear from the micrographs that the filament appearance is repeated every 90 degrees.

The distribution of filament widths for the single- and the double twisted structures are illustrated in figure 2 , for different nanotube loading fractions of $0.02 \%$ and $0.2 \%$ by weight. It can be seen that the width distributions are slightly asymmetric in nature, with a small filament width cut-off size. This behaviour is quite often observed in the growth of fibres, filaments or polymer strands, and can be described by a log-normal distribution, rather than a Gaussian distribution[23], which in this case is possibly related to curvature elasticity. For a liquid crystal a more slowly varying director field, i.e. smaller deformations per unit length, are energetically favourable.
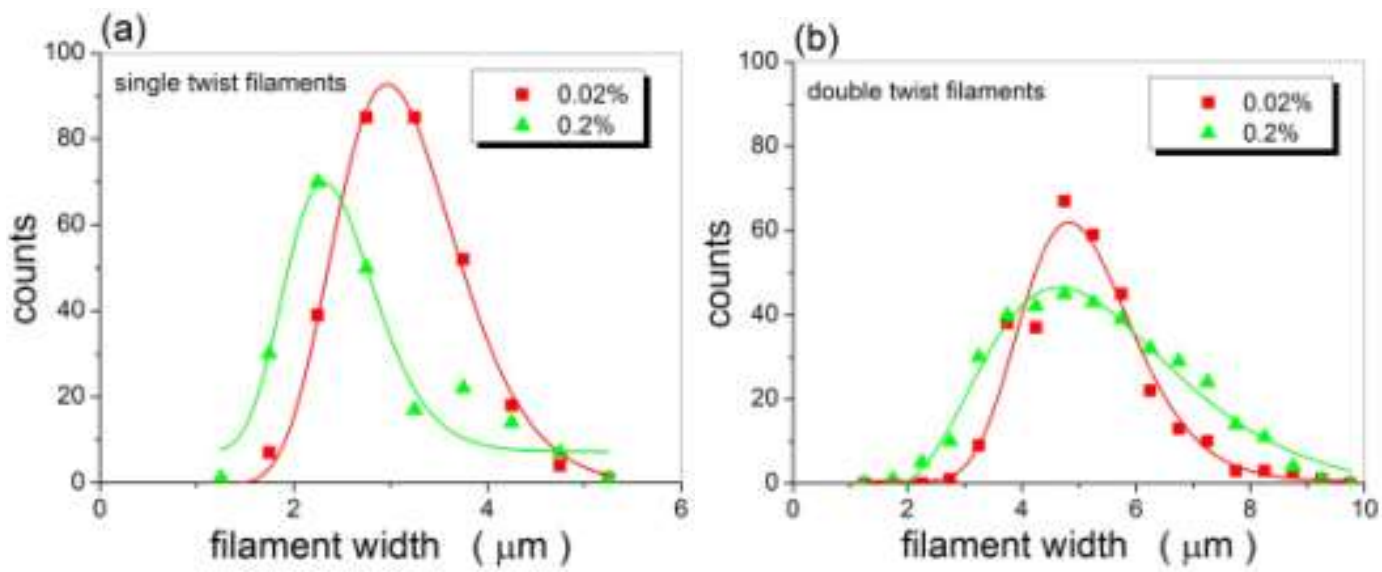

Figure 2: Filament width distributions for (a) single twist and (b) double twist filaments, for two selected nanotube concentrations of $0.02 \%$ and $0.2 \%$ by weight. Solid lines are a fit to a log-normal distribution, which describes asymmetries often observed in the growth of fibres, polymers or filaments.

The actual average filament width as a function of nanotube concentration is shown in figure 3 for both the single- and the double-twisted filaments. Both are practically constant as soon as nanotubes are added. Only for the non-doped samples of $0 \%$ nanotube concentration are the filament widths slightly larger. This could be due to a somewhat tighter binding of the filament to the nanotube bundles, because of the $\pi-\pi$ binding of the 
phenyl rings with the local hexagonal structure of the tubes, which is obviously absent in the non-doped samples, and the elastic behaviour of the liquid crystal.

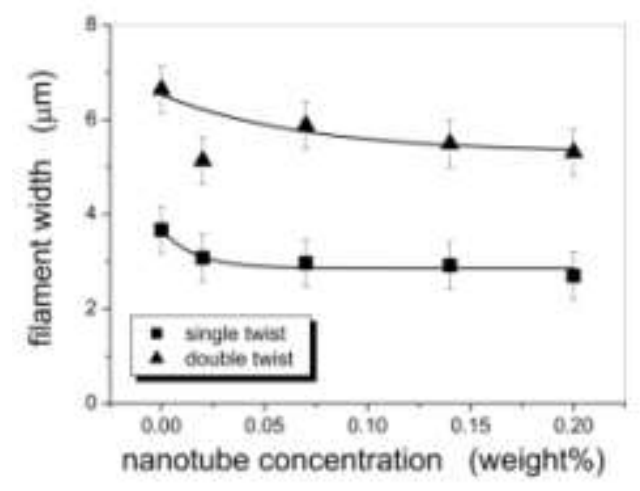

Figure 3: Average width of the single and double twisted filaments as a function of increasing nanotube concentration. The width slightly decreases when compared to the nondoped sample, before reaching saturation at larger nanotube concentrations.

The single-twisted spiralling structure is clearly tighter than the double-twisted one, and slightly larger than half the thickness of the latter. In both cases, one can observe a slight, yet not clearly pronounced, decrease in filament width as nanotubes / nanotube bundles are added to the liquid crystal with saturation quickly being reached for concentrations of approximately larger than $0.05 \%$ by weight. It is likely that the added nanotubes act like nucleation sites, with the helical filament structures forming around them, binding tighter to the nanotubes due to the largely enhanced $\pi$ - $\pi$-interactions between the nanotube and the aromatic core of the liquid crystal molecule. The nanotubes thus take the role of the core of a B7 filament, as shown schematically in figure 4. It should be noted that the structure of figure 4 is for illustrative purposes only, and does not reflect the actual sizes. The nanotube centre at the core of the filament is in reality a bundle of many single wall nanotubes held together by van der Waals interactions. The chirality of the real individual single wall tubes, as well as potentially that of the bundles, has no influence on the chirality of the filament, neither in magnitude (pitch) or sign (handedness). The bundles of nanotubes should rather be interpreted as cylinder-like filament cores that act a nucleation sites, with the by far most major contribution between the tubes and the liquid crystal being that of $\pi$ - $\pi$-interactions. 


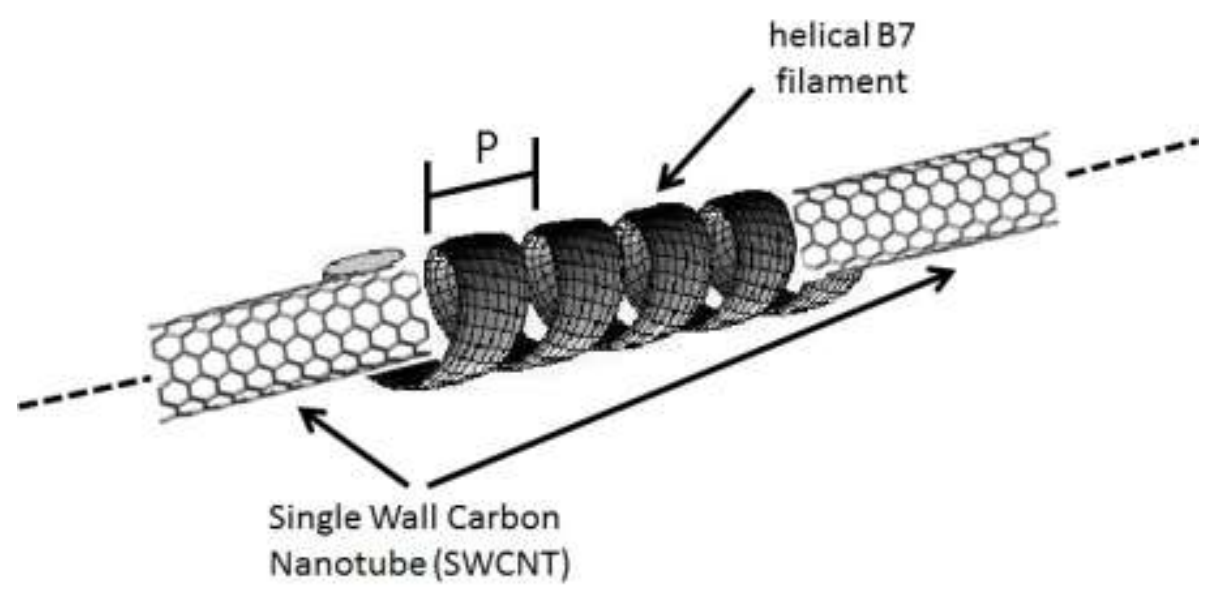

Figure 4: Schematic, not to scale, illustration of the nanotube acting a nucleation seed and core of the helical B7 filament during growth. The $\pi$ - $\pi$-interaction between the nanotube and the aromatic cores of the mesogens causes a tighter binding of the filament helix, while the pitch $P$ of the structure is not influenced.

Given the fact that the nanotubes generally cluster in bundles of diameters of several tens of nanometers, and that the chirality of the individual tubes is very small and of arbitrary handedness, we do not believe that the helical pitch of the filaments is in any way related to the helicity of the individual single wall nanotubes. This is also evidenced by the fact that the periodicity of the helix is not affected by the addition of nanotubes, as depicted in figure 5 . This is to be expected, because the nanotube chirality exerts an only very small contribution to the overall chirality of the system and individual tubes exhibit different and opposing chiralities, which cancels when viewed for the overall nanotube bundles consisting of many individual SWNTs.

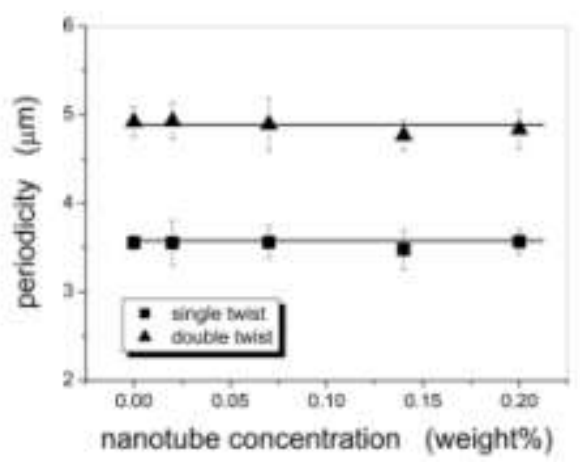


Figure 5: The periodicity of the twist is not affected by the addition of nanotubes, neither for the single nor the double twisted filaments. The periodicity of the double twisted helical superstructure is less than twice that of the single twisted filament.

It should be noted that even though the periodicity is independent of nanotube concentration for both types of twisted filament structures, the pitch of the double-twisted helix is considerable smaller than twice that of the single-twisted one.

An exemplary behaviour of the growth distance $d$ as a function of time $t$ is displayed for several nanotube concentrations in the case of (a) single-twisted and (b) double-twisted filaments of figure 6 . From the figures it is clear that in both cases the growth of the filaments is achieved in a largely linear $d(t)$ fashion, which allows the straight forward determination of the growth speeds as a function of nanotube concentration, $v=\delta d / \delta t=$ const. It should be pointed out though, that this linear behaviour is obviously only observed for undisturbed filament growth. If a growing filament comes in contact with another, or a different part of the structures formed during the phase transition, growth deviates from its linearity or ceases completely, in a process known as pinning. Also, when a filament nucleates from another, or both ends of a filament are fixed, the situation changes considerably, as will be discussed further below.
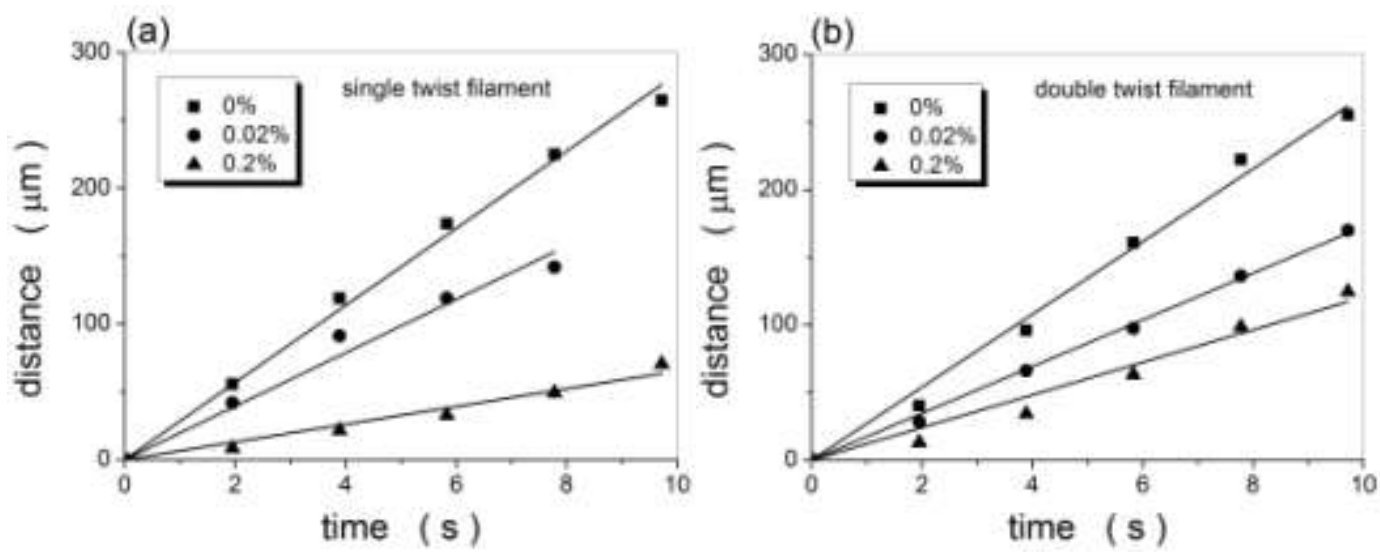

Figure 6: Exemplary demonstration of the linear filament growth observed for both (a) single and (b) double twisted helices, for several concentrations of nanotubes. The curves allow an easy determination of the growth speed of filaments. 
From a complete set of experimental data in accordance to figure 6 and averaging over approximately ten to fifteen independent measurements, the average growth speeds for the single- and the double-twisted filaments can be determined as a function of nanotube concentration. These are shown in figure 7 , with the error bars indicating the spread of the observed velocity values.
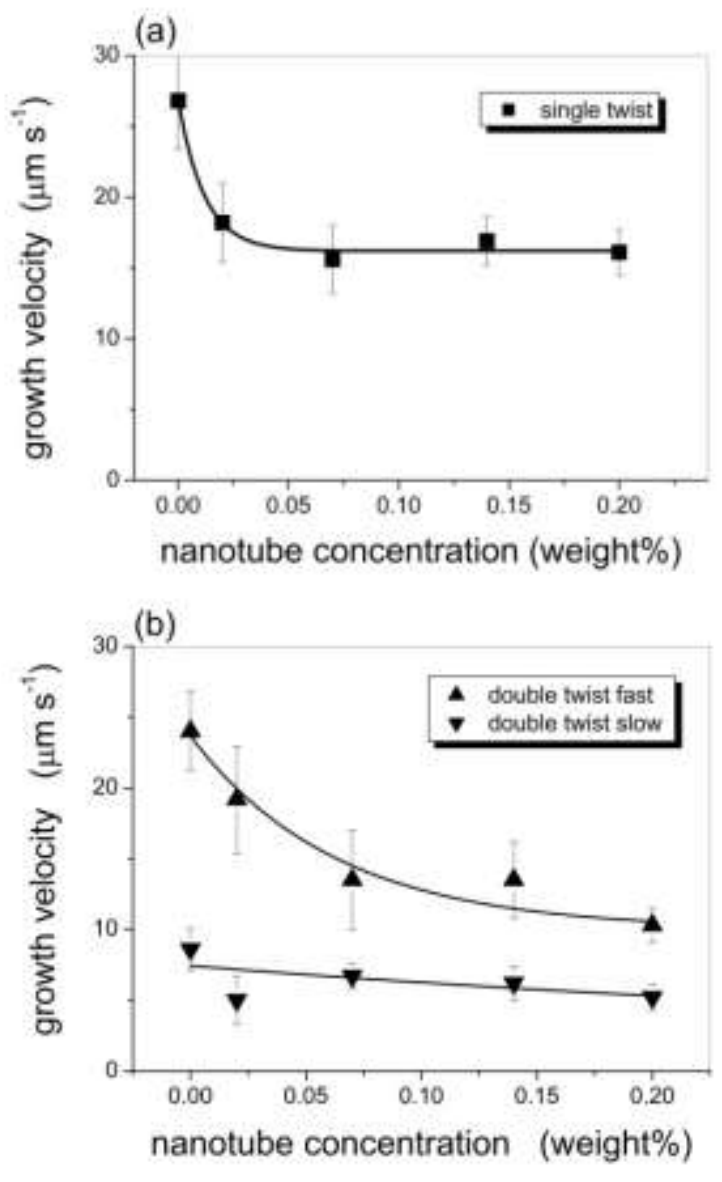

Figure 7: Growth velocities of (a) single and (b) double twist filaments as a function of nanotube concentration. Growth velocities decrease with increasing nanotube concentration until saturation, at which point presumable all filaments are affected by the dispersed nanotubes.

At this point it may be noted that the occurrence of single and double twisted filaments is not achieved with equal probability. In our samples, we observed about $25 \%$ of the filaments growing as single twisted structures, while approximately $75 \%$ were double twisted. The ratio of single to double twisted filaments is largely independent of nanotube 
addition, with no clear trend observed as a function of concentration. In contrast, the growth speed of the filaments decreases with increasing nanotube concentration for all structures observed. (fig. 7) until saturation at approximately $0.1 \%$ nanotubes by weight. It appears that at this nanotube concentration all filaments are influenced by the dopant, before other structures such as conformal domains and leaf-like structures can be observed to grow. The formation of tighter filaments with a smaller width for increasing nanotube concentration appears to be accomplished on a slightly longer time scale than for the nonor only lightly doped B7 material.

Further, when determining the growth velocity of different double twisted filaments at equal experimental conditions, it was found that the velocity distribution from approximately 15 independent speed measurements at each nanotube concentration, appeared to be bimodal with a clustering around lower and higher velocities observed (fig. 7(b)). This behaviour was increasingly pronounced for decreasing nanotube concentrations.

It should be pointed out that the here observed behaviour is different from that of filaments growing without any added nanotubes, as reported by Jakli et al.[24]. They observed a filament growth velocity which was approximately reciprocal to the filament diameter at nanotube concentrations of $0 \%$. The filament width was varied through a change in cooling rate, thus a change in the free energy, which implies non-equilibrium thermodynamic conditions. This case was not investigated in the present study. In our case we observed filament growth at a constant quench depth in thermodynamic equilibrium. Furthermore, we changed the weight concentration of dispersed nanotubes. From finding the filament diameter and the growth velocity slightly decreasing with concentration, our investigation would suggest a dependence of the filament growth velocity being roughly proportional to the filament width, $v \sim d$, instead of $v \sim 1 / d$ reported in [24]. We believe that the different behaviour is due to a varied experimental situation and parameterization, using doped versus non-doped samples, and thermodynamic equilibrium versus a non-equilibrium system. One would thus not expect to be able to carry out a direct comparison of the obtained results. 


\subsection{Filament buckling}

As pointed out above, a qualitatively different behaviour may be observed when the filaments are attached in either one or two points to other filaments, instead of simply nucleating and growing. In the first case, it is often observed that the filament grows in a linear fashion until after a certain length / time a buckling-phenomena, similar to static Euler elastic buckling for rigid bodies or Kirchhoff buckling in the flexible dynamic case, is initiated, as depicted in the growth time series of figure 8 . While the speed of the filament tip resembles that of an independent filament growing (fig. 7), the filament itself, at positions further back, forms a wavy, buckling instability, as it is known from compressive forces by viscosity on flexible filaments growing in viscous fluids $[25,26]$. Other classic examples have been theoretically described for telephone cables[27], but also for filament-like molecular structures such as macromolecules[28-31] of DNA or bacterial macrofibres, as pointed out in the theoretical review by Goriely and Tabor[32] . In the here observed buckling instability, the amplitude increases with the distance from the tip of the filament, while the instability wavelength is constant, $\lambda=(34 \pm 2.3) \mu \mathrm{m}$, when averaged over six periods under constant external conditions (figure 9).

Much of the work reported on buckling is theoretical or calculational in nature, often done in nonlinear dynamics, related to elasticity and engineering problems and thus performed by applied mathematicians. A scenario which appears to be of direct relevance to the present filament growth are the experimental investigations reported in reference [30]. The authors present results on metallic wires and rubber rods, subjected to a torsional buckling instability, which leads to observations similar to those depicted in fig. 8. Further twisting leads to post-buckling into a helix, which can be observed in fig. 10, when two filaments wrap around each other. This is the transition that in ref.[24] is introduced for beaded to supercoiled filaments. Another relevant topic related to the reported filament growth and the theoretical descriptions referenced above is the formation of natural fibres such as cellulose, tendrils or spider silk[33] and artificial fibres in the textiles industry[34].Very similar descriptions can account for systems at a much smaller length scale, for example the growth of bacterial macrofibres as discussed by Tilby[35] for the Bacillus subtilis, where backfolding and supercoiling can be observed. 

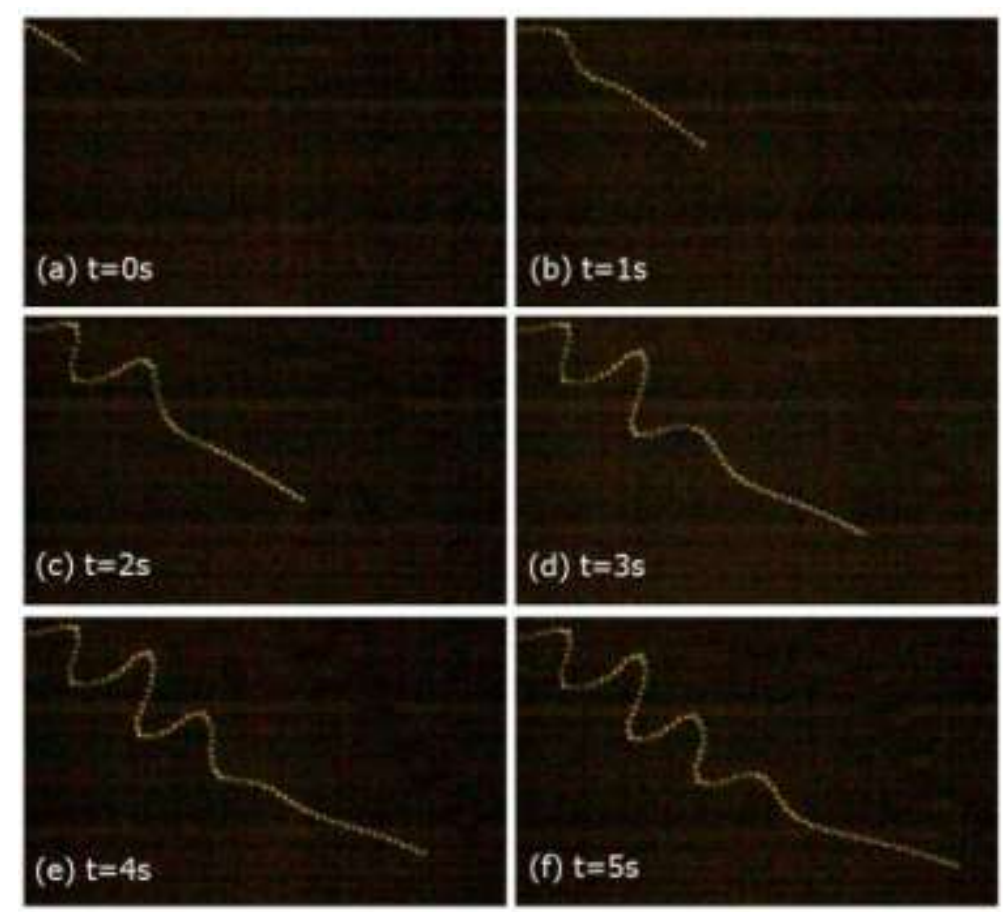

Figure 8: (a)-(f) time development of an elastic buckling instability for a filament which is fixed at its starting point of growth at another filament.

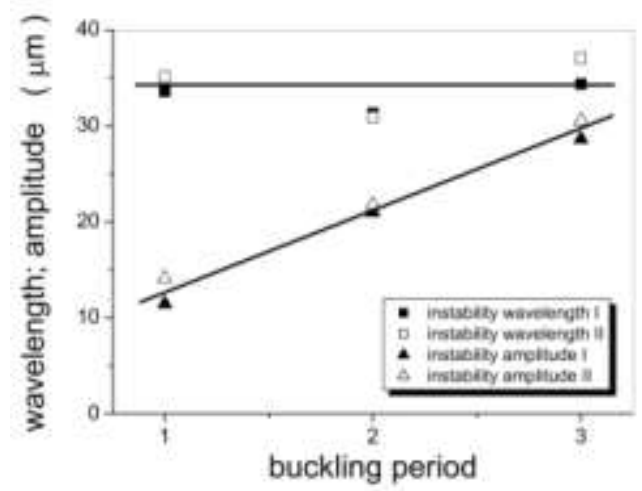

Figure 9: The amplitude (triangles) of the buckling instability increases with increasing period number, when moving away from the tip of the filament, while the wavelength of the instability (squares) is constant, as shown for two independent measurements (open and closed symbols).

In figure 10 the filament buckling is depicted for the case of the B7 filament being fixed at the two end positions. This situation is in principle similar to the one discussed above for a single fixture point and the development of a buckling pattern, only that here the pattern is more or less arbitrary. While a filament nucleating or being fixed in one point appears to 
grow through addition of the low temperature B7 phase material at the tip of the filament, and displays a very similar growth velocity in both cases, the filaments that are fixed on both ends appear to grow from both fixture points, as the observed growth velocity is largely constant and approximately twice as large as for the former case (figure 11).
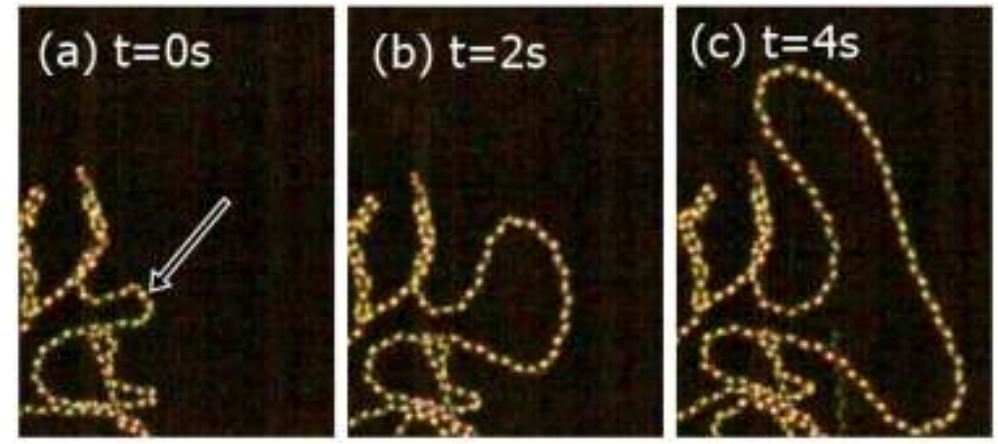

Figure 10: Growth of a filament (arrow in part (a)) which is fixed at the two end points. The buckling is caused for the same reasons as the instabilities of figure 8, only without displaying the regular instability pattern.

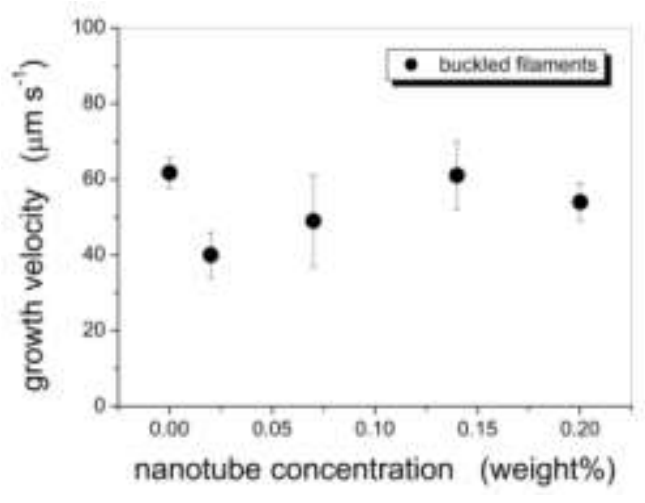

Figure 11: Nanotube concentration dependence of the growth velocity for filaments being fixed at both end points.

The buckling instability and its dynamics is qualitatively very similar to the experiment and theoretical discussion given by Vella et al.[36] for chains of magnetic spheres subjected to mechanical loading. Whereas in the magnetic chain case the buckling is due to pushing the fixed two endpoints of the chain closer together, it is in the case of nanotube doped B7 filaments the progressing volume production of B7 material between constant fixed filament endpoints. 


\section{Conclusions}

We have investigated the effect of dispersed single wall carbon nanotubes on the growth of bent-core B7 filaments at the transition from the isotropic liquid to the liquid crystalline state. Different types of helical filaments can be identified, together with conformal domains and leaf-like structures. For both single twist and double twist filaments, the average width of the filaments is slightly decreasing with increasing nanotube content. This behaviour suggests that the nanotubes act as nucleation sites for the filaments, which wrap around the tubes and take the role of the filament core. The periodicity of the helical structure is not affected, which demonstrates that the intrinsic nanotube chirality is very weak and not sufficiently strong to overcome the steric interactions of the bent-core molecules, which leads to the formation of the left- and right-handed helical superstructures. At the same time, the growth velocity of the filaments decreases for increasing nanotube concentration, saturating at the same SWCNT concentration at which also the change in filament diameter ceases. While for single twisted filaments only one most probable filament velocity is observed, velocity values for the double twisted filaments cluster around two values, slow and fast, which are clearly different for all nanotube concentrations, and for which the fast growth velocity is approximately equal to that of the single twist filaments.

Additionally, an elastic buckling instability was observed, which for filaments fixed on one end, shows a constant period and increasing amplitude as one proceeds away from the filament tip, i.e. in opposite direction as filament growth. Fixing both ends of a growing filament results in an increased filament growth velocity and a buckled structure, though without the well defined periodicity of its single fixed counterpart. The richness of the observed behaviour suggests that the discussed system may be an interesting experimental ground to study and investigate theoretical models of nonlinear dynamics of filaments, also in comparison to similar scenarios in different experimental systems as mentioned above.

Besides the more fundamental understanding of experimental nano- and micro-systems for nonlinear dynamics, B7 filament - nanotube dispersions may be used as model systems in 
biomolecular ordering of DNA or bacterial macrofibres, in the directed growth of switchable optical structures, or for the generation of high tensile strength fibres, combining liquid crystal ordering (like Kevlar) with the extraordinary mechanical properties of nanotubes.

Acknowledgements:

O.S. would gratefully like to acknowledge the financial support of the Erasmus Program of the European Union for a traineeship to carry out this research.

\section{References}

[1] P.J. Collings, M. Hird, Introduction to Liquid Crystals: Chemistry and Physics, Taylor\&Francis, London, 1997.

[2] S. Chandrasekhar, Liquid Crystals, $2^{\text {nd }}$ ed., Cambridge University Press, Cambridge, 1992.

[3] P.G. de Gennes, J. Prost, The Physics of Liquid Crystals, $2^{\text {nd }}$ ed., Clarendon Press, Oxford, 1993.

[4] T. Niori, T. Sekine, J. Watanabe, T. Furukawa, H. Takezoe, J. Mater. Chem., 1996, 6, 1231.

[5] G. Pelzl, S. Diele, W. Weissflog, Adv. Mater., 1999, 11, 707.

[6] H. Takezoe, Y. Takanishi, Jpn. J. Appl. Phys. A, 2006, 45, 597.

[7] H. Takezoe, A. Eremin, Bent-shaped Liquid Crystals, CRC Press, Taylor\&Francis Group, Boca Raton, 2017.

[8] D.R. Link, G. Natale, R. Shao, J.E. Maclennan, N.A. Clark, E. Korblova, D.M. Walba, Science, 1997, 278, 1924.

[9] J. Thisayukta, H. Niwano, H. Takezoe, J. Watanabe, J. Am. Chem. Soc., 2002, 124, 3354. 
[10] E. Gorecka, M. Cepic, J. Mieczkowski, M. Nakata, H. Takezoe, B. Zeks, Phys. Rev. E, 2003, 67, 061704.

[11] P. Archer, I. Dierking, ChemComm., 2010, 46, 1467.

[12] G. Pelzl, S. Diele, A. Jakli, C. Lischka, I. Wirth, W. Weissflog, Liq. Cryst., 1999, 26, 135.

[13] G. Pelzl, S. Diele, A. Jakli, W. Weissflog, Liq. Cryst., 2006, 33, 1513.

[14] A. Jakli, D. Krüerke, G.G. Nair, Phys. Rev. E, 2003, 67, 051702.

[15] R. Deb, M. Oneill, N.V.S. Rao, N.A. Clark, I.I. Smalyukh, ChemPhysChem, 2015, 16, 243.

[16] D.K. Yoon, R. Deb, D. Chen, E. Korblova, R. Shao, K. Ishikawa, N.V.S. Rao, D.M. Walba, I.I. Smalyukh, N.A. Clark, PNAS, 2010, 107, 21311.

[17] D. Chen, D.K. Yoon, J.E. Maclennan, M.A. Glaser, E. Korblova, D.M. Walba, N. Gimeno, M.B. Ros, R. Deb, N.V.S. Rao, N.A. Clark, Soft Matter, 2013, 9, 11303.

[18] C. Zhang, S.M. Salini, N. Diorio, W. Weissflog, A. Jakli, Liq. Cryst., 2015, 42, 1621.

[19] J.P.F. Lagerwall, G. Scalia (eds.), Liquid Crystals with Nano- and Micro-Particles, World Scientific Publishing, Singapore, 2017, and references therein.

[20] I. Dierking, G. Scalia, P. Morales, D. LeClere, Adv. Mater., 2004, 16, 865.

[21] I. Dierking, G. Scalia, P. Morales, J. Appl. Phys., 2005, 97, 044309.

[22] G. Heppke, D.D. Parghi, H. Sawade, Liq. Cryst., 2000, 27, 313.

[23] C.J. Ellison, A. Phatak, D.W. Giles, C.W. Macosko, F.S. Bates, Polymer, 2007, 48, 3306.

[24] A. Jakli, C. Lischka, W. Weissflog, G. Pelzl, A. Saupe, Liq. Cryst., 2000, 27, 1405.

[25] B. Audoly, Y. Pomeau, Elasticity and Geometry: From hair curls to the non-linear response of shells, Oxford University Press, Oxford, 2010.

[26] M. Le Merrer, D. Quere, C. Clanet, Phys. Rev. Lett., 2012, 109, 064502.

[27] J. Coyne, IEE Journal of Oceanic Engineering, 1990, 15, 72. 
[28] M.D. Barkley, B.H. Zimm, J. Chem. Phys., 1979, 70, 2991.

[29] N.H. Mendelson, Sci. Prog. Oxford, 1990, 74, 425.

[30] J.M.T. Thompson, A.R. Champneys, Proc. Royal Soc. London Series A, 1996, 452, 117.

[31] T. Schlick, Cur. Opinions Struct. Biol., 1995, 5, 245.

[32] A. Goriely, M. Tabor, Nonlinear Dynamics, 2000, 21, 101.

[33] J.P. Canejo, M.H. Godinho, Materials, 2013, 6, 1377.

[34] J.W.S. Hearle, J.J. Thwaites, J. Amirbayat (eds.), Mechanics of Flexible Fibre Assemblies, Sijthoff \& Noordhoff, Germantown, 1980.

[35] M. J. Tilby, Nature, 1977, 266, 450.

[36] D. Vella, E. du Pontavice, C.L. Hall, A. Goriely, Proc. Royal Soc. London Series A, 2014, 470, 20130609. 